\title{
Closed loop system on the base of non-invasive optical glucometer
}

\begin{abstract}
Closed loop system for diabetes mellitus compensation was developed. The system comprises a non-invasive optical glucose meter, an insulin infusion device and algorithm for blood glucose prediction. Glucometer consists of the control unit connected to the earlap clip by optical fiber. Experimental trials show that inherent accuracy of the optical system is $0.012 \%$, reproducibility $-0.06 \%$, RMSE in the blood glucose physiological area $-17 \%$. The system was provided with the short-term prediction algorithm, which corrects $96.5 \%$ cases of inaccurate operation of the glucometer that makes insulin infusion more physiological. The system is aimed for personal continuous blood glucose control for patients with diabetes mellitus type I.
\end{abstract}

Keywords: non-invasive glucose meter, artificial pancreas, optical, diabetes mellitus
Volume 3 Issue 7 - 2016

\author{
Nikolay A Bazaev, Kirill V Pozhar, Evgeniia L \\ Litinskaia \\ Department of Biomedical Engineering, National Research \\ University of Electronic Technology, Russia
}

Correspondence: Nikolay A Bazev, Department of Biomedical Engineering, National Research University of Electronic Technology Zelenograd, Moscow, Russia, Email bazaev-na@yandex.ru

Received: July 28, 2016 | Published: December 06, 2016

\section{Introduction}

Diabetes mellitus is the third widespread lethal disease in the world. It is an endocrine disease, characterized by chronic increase of blood glucose concentration (BG) that occurs when pancreas is no longer available to produce enough amount of insulin. The potentially breakthrough method of diabetes mellitus therapy is the continuous closed loop blood glucose control system, ${ }^{1}$ which is aimed to compensate carbohydrate metabolic imbalance by continuous insulin infusion. To provide adequate insulin therapy it is required to analyze $\mathrm{BG}$, which can be measured by portable blood glucose meters or glucose monitors. Up-to-date commercial BG monitoring systems and glucometers are invasive. ${ }^{2}$ Their application brings such disadvantages as discomfort and risk of infection. Development of a non-invasive system for continuous blood glucose monitoring seems to be a very promising direction. Such a BG monitor can work continuously and provide user with BG dynamics during a long period of time that is necessary for accurate diabetes mellitus compensation. Combination of non-invasive continuous blood glucose monitor with automatic insulin pump gives a possibility for on-line insulin infusion rate correction. Such a system must be provided with algorithms for interaction between it parts and short-term BG prediction. The latter is to predict glucose and insulin dynamics and estimate adequacy of glucometer data. Thus, possible glucometer errors or problems with tubing set bending etc. could be avoided. In current paper we present results of our development of a closed loop system on the base of noninvasive optical glucometer.

\section{Methodology}

\section{Non-invasive glucometer}

Portable non-invasive glucose meter implement transmission spectroscopy method. Infrared radiation from laser light source spreads through tissues and reaches photodiode on the outer side. The glucometer consists of control unit and clip for earlap (Figure 1). The light source is a NIR laser photodiode with thermos-stabilization unit. The clip contains optical system and photodiode. To avoid clip heating and to reduce clip size light source is placed in the control unit, which is connected to the clip by optical fiber. The calculated blood glucose level is displayed on the display panel, saved and can be transmitted to $\mathrm{PC}$ or smartphone if necessary. Control unit can be connected to PC by USB and interacts with insulin pump by Bluetooth. The device powering is provided by PP3-size battery or by an AC-adapter. Calculation of blood glucose level is based on the iteration algorithm taking into consideration reference data.

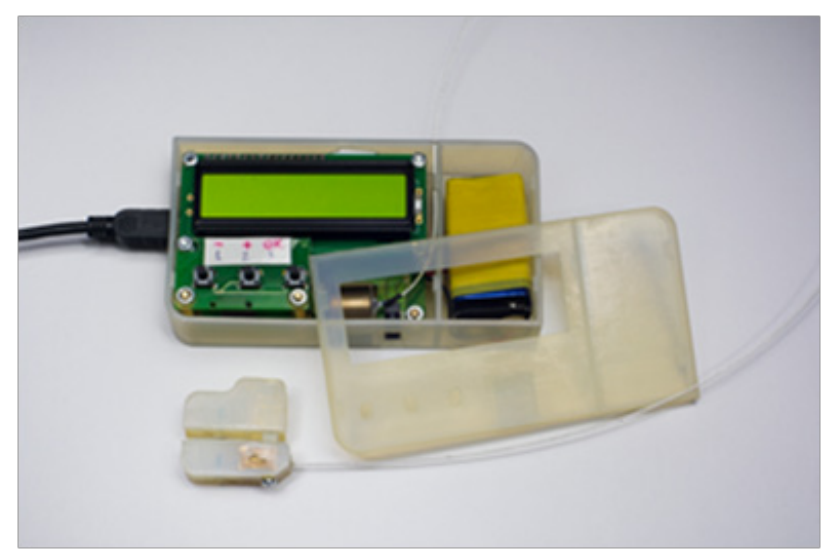

Figure I Non-invasive portable optical glucometer (prototype).

\section{Insulin pump}

Insulin delivery system is an automatic insulin pump with stepper motor and $3 \mathrm{ml}$ insulin reservoir. The device powering is provided by AA-size rechargeable battery. The pump control unit has Bluetooth interface for data exchange with glucose meter control unit and smart phone. Smartphone application is based on user interface comprising the following functions: food intake and other factors affecting on BG dynamics data input; calibration data input; current BG and dynamics diagram visualization; error indication (Figure 2).

\section{Prediction algorithm}

To predict BG it is necessary to have approbated on real BG tracks mathematic models of glucose and insulin regulation. Developed mathematical model takes into consideration a range of factors affecting the blood glucose dynamics ${ }^{3}$ and mechanisms of blood glucose and insulin regulation ${ }^{4}$ in human body. Developed sigmamodel $^{4}$ is used as the basis of short-term prediction algorithm ${ }^{5}$ that 
is to detect errors in operating of pump or glucometer. The model predicts the blood glucose level during next 30 minutes on the base of previous data from patient and information about meals and insulin injections. If glucometer indications differ from predicted by more than $20 \%$ the indication is considered as an error. Otherwise the glucose meter indication is considered as correct.

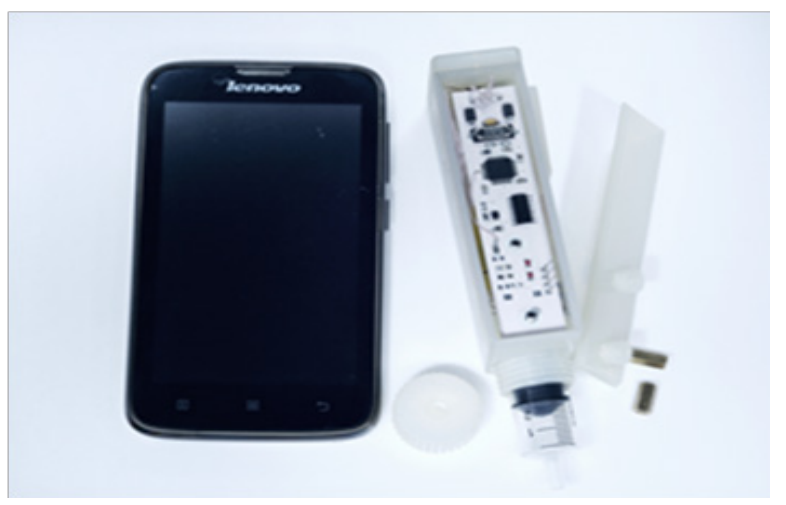

Figure 2 Insulin Pump Prototype.

\section{Experimental trials}

Adequacy of developed glucometer was estimated via Clarke Error Grid Analysis (Figure 3). None of indications falls in E-zone of Clarke error grid, therefore the usage of the device can't cause heavy health hazard. Inherent accuracy of the device optical system is $0,012 \%$, reproducibility of measurement results $-0,06 \%$. Mean error of measurements in the blood glucose physiological area is $17 \%$. Prediction algorithm approbation showed that $96.5 \%$ cases of incorrect operation of the BGC monitor was corrected that confirms reliability of the combined system. Approbation was carried out on the basis of glucose tracks database DirecNet. ${ }^{6}$

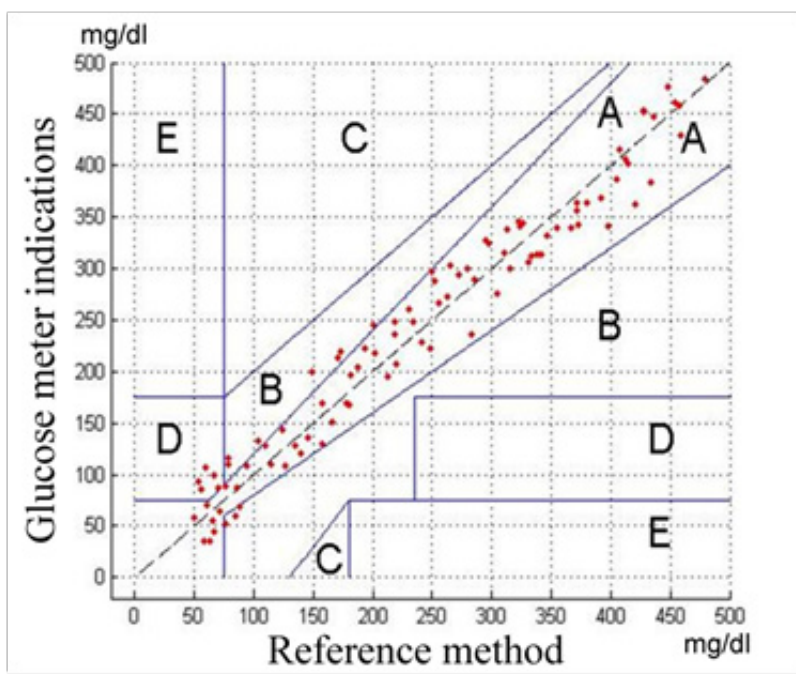

Figure 3 Non-invasive portable optical glucometer prototype measurements on Clarke Error Grid.

\section{Results and discussion}

Results of approbation of non-invasive glucometer and algorithms for blood glucose and insulin regulation and prediction gives us optimism for further steps toward development of reliable closed loop system for diabetes compensation on the base of an optical noninvasive glucometer. The system will be applicable for constant blood glucose control for patients with diabetes mellitus type I.

\section{Acknowledgements}

None.

\section{Conflict of interest}

Author declares that there is no conflict of interest.

\section{References}

1. Cobelli C, Renard E, Kovatchev B. Artificial Pancreas: Past, Present, Future. Diabetes. 2011;60(11):2672-2682.

2. Agrawal P, Welsh JB, Kannard B, et al. Usage and Effectiveness of the Low Glucose Suspend Feature of the Medtronic Paradigm Veo Insulin Pump. J Diabetes Sci Technol. 2011;5(5):1137-1141.

3. Bazaev NA, Pozhar KV, Pletenev AN. Classification of Factors Affecting Blood Glucose Concentration Dynamics. Biomedical Engineering. 2013;47(2):100-103.

4. Bazaev NA, Pozhar KV, Rudenko PA. Mathematical modeling of Blood Glucose Concentration Dynamics. Biomedical Engineering. 2015;48(6):292-296.

5. Bazaev NA, Pozhar KV, Pletenev AN. An Algorithm for Short-Term Prediction of Blood Glucose Concentration. Biomedical Engineering. 2014;47(6):312-314.

6. Ruedy KJ, Beck RW, Xing D, et al. Diabetes Research in Children Network: Availability of Protocol Data Sets. J Diabetes Sci Technol. 2007;1(5):738-745. 